\title{
Migration und Entwicklung : Fragen zu einer umstrittenen Beziehung
}

Denise Efionayi-Mäder, Gérard Perroulaz and Catherine Schümperli Younossian

\section{(2) OpenEdition}

12 Journals

\section{Electronic version}

URL: http://journals.openedition.org/sjep/303

DOI: 10.4000/sjep.303

ISSN: 1663-9677

\section{Publisher}

Institut de hautes études internationales et du développement

\section{Printed version}

Date of publication: 1 décembre 2008

Number of pages: 11-21

ISBN: 978-2-940415-08-3

ISSN: $1660-5926$

\section{Electronic reference}

Denise Efionayi-Mäder, Gérard Perroulaz und Catherine Schümperli Younossian, « Migration und Entwicklung : Fragen zu einer umstrittenen Beziehung », Schweizerisches Jahrbuch für

Entwicklungspolitik [Online], 27-2 | 2008, Online erschienen am: 25 Mai 2010, abgerufen am 25 September 2020. URL : http://journals.openedition.org/sjep/303 ; DOI : https://doi.org/10.4000/sjep. 303 


\title{
Migration und Entwicklung: Fragen zu einer umstrittenen Beziehung
}

\author{
Denise Efionayi-Mäder, Gérard Perroulaz \\ und Catherine Schümperli Younossian*
}

\begin{abstract}
S
eit rund dreissig Jahren haben sich die internationalen Migrationsbewegungen weltweit intensiviert und sind komplexer geworden. Die Migration geht mit einer zunehmenden Mobilität von Waren, Dienstleistungen, Kapital und Information auf regionaler wie auf interkontinentaler Ebene einher. Angesichts der weltweit wachsenden Ungleichheiten ist das Migrationsphänomen fortan untrennbar mit Fragen der Entwicklung der Länder des Südens verbunden. Zum Einkommensgefälle innerhalb der Länder wie auch zwischen den Staaten kommen die sozialen, rechtlichen und gesundheitlichen Unterschiede hinzu.
\end{abstract}

Schätzungen zufolge leben in der Welt etwa 200 Millionen Menschen mindestens ein Jahr lang nicht in ihrem Geburtsland ${ }^{1}$; fast die Hälfte davon sind Frauen. In der Schweiz ist eine von drei Personen ausländischer Herkunft durch ihre Eltern, und ein Viertel der erwerbstätigen Bevölkerung besitzt keinen Schweizer Pass. Diese Personen sind zum einen in Arbeitsbereichen tätig, die von den Einheimischen gemieden werden, und bringen zum anderen auf gefragte, berufliche Qualifikationen mit.

Die Integration von Ausländerinnen und Ausländern und die irreguläre Zuwanderung nehmen heute einen bedeutenden Platz in den öffentlichen Debatten ein und kristallisieren die politischen Gegensätze heraus. Migration darf aber nicht nur auf diese Aspekte beschränkt werden; sie steht auch für Wissensvermittlung und Erfahrungsaustausch, für kulturelle Beiträge und für Entwicklung in sämtlichen

* Denise Efionayi-Mäder, Projektleiterin und Vize-Direktorin des Schweizerischen Forums für Migrations- und Bevölkerungsstudien (SFM), Universität Neuenburg.

Gérard Perroulaz, Forschungsbeauftragter am Institut de hautes études internationales et du développement (IHEID), Genf.

Catherine Schümperli Younossian, Leiterin des Redaktionskomitees des Schweizerischen Jahrbuchs für Entwicklungspolitik und Lehrbeauftragte am Institut de hautes études internationales et du développement, Genf.

Das Dossier 2008 ist das Ergebnis einer fruchtbaren Zusammenarbeit zwischen dem Institut de hautes études internationales et du développement (IHEID) in Genf und dem Schweizerischen Forum für Migrations- und Bevölkerungsstudien (SFM) der Universität Neuenburg. Mittels der Netzwerke beider Institutionen konnten massgebliche Autorinnen und Autoren aus Akademikerkreisen, internationalen Organisationen, aus der Bundesverwaltung und aus Nichtregierungsorganisationen ermittelt werden. Das Redaktionskomitee des Schweizerischen Jahrbuchs für Entwicklungspolitik dankt Denise Efionayi-Mäder und Alessandro Monsutti, Dozent am IHEID, sehr herzlich für ihre unermüdliche Unterstützung bei der Gestaltung und Durchführung dieses Werks, wodurch die wissenschaftliche Gültigkeit der ausgewählten Beiträge gewährleistet wird. Wir danken auch den Evaluatoren der Artikel, darunter Reynald Blion, Gianni D’Amato und Etienne Piguet.

1 Gemäss der Definition der Vereinten Nationen ist ein Migrant oder eine Migrantin eine Person, die ihr Land für mindestens ein Jahr mit der Absicht verlässt, sich langfristig im Ausland niederzulassen. 
Ländern des Südens und des Nordens. Die Beziehungen zwischen Migration und Entwicklung sind schon seit langem Gegenstand der Forschung im Migrationsbereich gewesen, doch hat das Thema seit einiger Zeit wieder an Interesse gewonnen und ist auf die politische Agenda der Vereinten Nationen und der internationalen Organisationen gerückt. Die nationalen Kontroversen um die Migrationspolitik und die Politik der Entwicklungszusammenarbeit waren dabei ausschlaggebend.

Das vorliegende Dossier des Schweizerischen Jahrbuchs für Entwicklungspolitik hat sich zum Ziel gesetzt, die erörterten Themen aus dem Blickwinkel der akademischen Forschung und der Politikfeldanalyse auf nationaler und internationaler Ebene anzugehen. Fallstudien, Aussagen und Stellungnahmen tragen zur Veranschaulichung der theoretischen Artikel bei, um zum Nachdenken und zur Diskussion anzuregen.

In seinem einführenden Beitrag zum Dossier stellt Alessandro Monsutti die verschiedenen Theorieansätze der Sozialwissenschaften vor, die der Reflexion über die Beziehungen zwischen Migration und Entwicklung in der Nachkriegszeit zugrunde lagen. Der Autor zeigt auf, dass die Meinungen zwischen dem Optimismus der Modernisierungstheorie in den 1950er und 1960er Jahren und der Skepsis der Abhängigkeitstheorie in den 1970er und sogar in den 1980er Jahren schwankten. Es geht somit darum, die positive Einschätzung der Auswirkungen der Migration auf die Entwicklung des Südens - eine Einschätzung, die seit einigen Jahren auf der politischen Bühne zu überwiegen scheint - in die richtige Perspektive zu rücken. Das gegenwärtige Interesse für die Geldüberweisungen der Migranten lässt eine konstruktive Betrachtungsweise der Mobilität zu, jedoch besteht die Gefahr einer politischen Instrumentalisierung. Nach Ansicht des Autors lässt die Tendenz, die internationale Migration und die Rücküberweisungen pauschal als die neue Patentlösung gegen die Unterentwicklung der Länder des Südens preisen zu wollen, einen Mangel an historischer und theoretischer Perspektive erkennen. Während die Weltkommission für internationale Migration (World Commission on International Migration) die Notwendigkeit einer verstärkten zwischenstaatlichen Koordination betonte, sind die Staaten nicht gewillt, ihren Souveränitätsanspruch auf die Kontrolle der Ein- und Auswanderung in ihr Hoheitsgebiet preiszugeben, und bekunden Mühe, sich auf einen globalen Rahmen zur Bewältigung der Migrationsfrage zu einigen (die bestehenden Kooperationsabkommen betreffen im Wesentlichen Sicherheitsfragen).

Dennoch hat die Kenntnis vom Zusammenhang zwischen Migration und Entwicklung beträchtliche Fortschritte gemacht. Dank der zahlreichen Studien der letzten Jahre beginnt man, die Folgen der Migrationsbewegungen für die Herkunfts-, Transit und Aufnahmeländer zu kennen. Die Auswirkungen der Entwicklung auf die Migrations werden besser und in ihrer gesamten Vielschichtigkeit erfasst. Auch wenn die Entwicklungszusammenarbeit in einer ersten Phase eine mit armutsbedingter Abwanderung - zum Beispiel im Fall der Landflucht - verhüten kann, erhöht die Wirtschaftsentwicklung zuweilen den Migrationsdruck auf mittlere Sicht, bevor sie zu ihrem längerfristigen Rückgang beiträgt (migration hump). Wenngleich die Visionen der Entwicklungszusammenarbeit und der Migrationspolitik mittelfristig gesehen als gegensätzlich erscheinen mögen, haben diese unterschiedlichen Sichtweisen, aus einer langfristigen Perspektive heraus betrachtet, Tendenz, sich einander anzunähern. 
Die Beziehung zwischen Migration und Entwicklung hat sich aufgrund des wachsenden Interesses für die Geldtransfers oder Rücküberweisungen der Migranten - so genannte Remittances - und ihre Auswirkungen auf die lokale Wirtschaft der Herkunftsländer durchgesetzt. Die Untersuchung der Finanzflüsse zeigt, dass die von den Migranten überwiesenen Summen die von der öffentlichen Entwicklungshilfe aufgebrachten Beträge bei weitem übersteigen, und zwar ohne Berücksichtigung der informellen Geldflüsse und der Warenströme, die nicht unbeträchtlich sind. Für 2006 schätzt die Weltbank die Remittances auf 206 Milliarden Dollar, während sich die öffentliche Entwicklungshilfe der Mitgliedsländer der Organisation für wirtschaftliche Zuammenarbeit und Entwicklung (OECD) auf 104 Milliarden Dollar beläuft. Philippe Wanner weist jedoch auf die methodologischen Schwierigkeiten hin, die mit einer Definition des Konzepts der Remittances verbunden sind, und auf die Unzulänglichkeit der statistischen Daten, die Zurückhaltung bei der Interpretation der Zahlen gebieten. Über die quantitative Frage hinaus stellt sich der Autor Fragen zu zwei Aspekten, nämlich betreffend die Auswirkungen der Rücküberweisungen auf die Entwicklung der Länder des Südens und bezüglich der verschiedenen Überweisungsmethoden. Diese Fragen finden im Rahmen der hier präsentierten Fallstudien von Claude Auroi, Alessandro Monsutti und Matthias Lerch Ansätze einer Antwort.

Inwieweit ermöglichen es die Rücküberweisungen, die Armut zu bekämpfen und fördern sie produktive Investitionen in den Ländern des Südens? Wanner erinnert daran, dass sich die Literatur darauf beschränkt, die empirisch beobachteten Auswirkungen in den von der Emigration betroffenen Ländern zu beschreiben und zu interpretieren, ohne die anfallenden Kosten zu berücksichtigen. Der Autor anerkennt zwar, dass die Rücküberweisungen ein grosses Potenzial besitzen, stellt aber auch fest, dass sie in den ärmsten Ländern nicht immer optimal zur Entwicklung beitragen. Sie könnten sogar innerhalb der Empfängerländer der Gelder zu starken Ungleichheiten führen zwischen jenen, die in den Genuss von Rücküberweisungen kommen, und jenen, die nicht dieses Vorrecht haben. Wanner verweist darauf, dass den Begünstigten häufig vorgeworfen wird, nicht in Tätigkeiten zu investieren, die Arbeitsplätze und Wohlstand schaffen.

Claude Auroi untersucht die Beziehung zwischen den Geldüberweisungen der Migranten und der lokalen und regionalen Wirtschaftsentwicklung in Lateinamerika. Der Autor schätzt eingangs das Volumen der Geldtransfers nach Lateinamerika ein, präzisiert ihre relative Bedeutung für die Volkswirtschaft einiger Länder der Region und befasst sich dann mit ihrer Verwendung. Seine Beurteilung schliesst sich jener Wanners an: Die Ausgaben der Migrantenfamilien sind hauptsächlich Konsumausgaben. Jedoch relativiert der Autor diese negative Feststellung, indem er betont, dass die von den Migrantenfamilien beschlossenen Ausgabenstrategien als zukunftsträchtig gelten können; und er fügt hinzu: „Die Geldsendungen der Migration tragen zur Vorsorge und zur sozialen Sicherheit bei, welche der Staat in Lateinamerika nicht leisten kann.“

Wanner und Auroi weisen beide auf die Schwierigkeit hin, „private“ Initiativen von Geldsendungen in kollektive Projekte umzuleiten. Gleichzeitig anerkennen sie aber die Notwendigkeit, Anreizmassnahmen zu schaffen, um das Potenzial 
der Geldüberweisungen besser zu nutzen, nach dem Beispiel des Programms Tres por uno, das in Mexiko und El Salvador zur Anwendung kommt ${ }^{2}$.

Die Wahl der Überweisungsmethoden gibt über wichtige Aspekte der sozioökonomischen Lage der Migrantinnen und Migranten Aufschluss, woraus sich das die Durchführung zahlreicher Fallstudien erklärt, um die diesbezüglichen Mechanismen zu verstehen. Wanner verweist darauf, dass diese Mechanismen hauptsächlich von den Bankenstrukturen der Herkunftsländer und vor allem der Bestimmungsländer abhängen. In einigen Ländern des Südens besteht ein starker Mangel an Banken, insbesondere in den ländlichen Gebieten. Dies fördert das Aufkommen spezialisierter Finanzakteure wie von Western Union, die weltweit über 170000 Zweigstellen verfügt. Jedoch sind die von ihnen in Rechnung gestellten Transaktionskosten im Vergleich zu den überwiesenen Summen relativ hoch. Gemäss den Schätzungen des Autors werden in manchen Ländern bis zu 80 Prozent der Überweisungen weiterhin über informelle Kanäle abgewickelt. Zur Veranschaulichung dieser Tatsache werden zwei Fallstudien im Dossier präsentiert, die eine betreffend Rücküberweisungen nach Afghanistan (Alessandro Monsutti), die andere bezüglich Rücküberweisungen aus der Schweiz nach Serbien (Matthias Lerch).

\section{Vom Braindrain zur "Brain Circulation”}

Während sich die Debatten in den internationalen Organisationen weitgehend auf die Geldüberweisungen fokussierten, hat die Forschung ihre Aufmerksamkeit auch der Übermittlung von Kenntnissen - hauptsächlich unter dem Gesichtspunkt der Rekrutierung von Arbeitskräften und der Ausbildung gewidmet. Auch wenn die Abwanderung qualifizierter Arbeitskräfte in die Länder des Nordens eine Realität ist, die vor allem die ärmsten Länder betrifft, können die Migration von Fachkräften und die Internationalisierung ihrer Ausbildung sowohl für die Herkunfts- als auch für die Aufnahmeländer eine Chance darstellen. Jean-Baptiste Meyer beschreibt das Aufkommen transnationaler wissenschaftlicher Diasporas ${ }^{3}$, welche die Süd-Nord-Ströme hoch qualifizierter Fachkräfte beschränken können. Diese diasporischen Wissensnetzwerke (diaspora knowledge networks) sind in der Regel assoziativ organisiert und können vielfältige - sowohl virtuelle als auch konkrete - Formen annehmen. Ihre Tätigkeiten reichen vom fachlichen Informationsaustausch über das Tutoring von fortgeschrittenen Studierenden und die punktuelle Beschäftigung von Expatriierten an Universitäten ihrer Herkunftsländer bis zur Durchführung gemeinsamer Entwicklungsprojekte. Die verfügbaren empirischen Daten zeigen, dass ein Grossteil der hoch qualifizierten Migranten den Herkunftsländern ihr Fachwissen zur Verfügung stellen wollen. Wenn Indien und China an der Spitze der im Internet erfassten diasporischen Wissensnetzwerke stehen, die sich für die Entwicklung ihrer Länder einsetzen, so hat das Phänomen auch weltweit ein erhebliches Ausmass erreicht.

2 Für jeden Dollar, der von den Migranten in gemeinnützige Projekte investiert wird, werden von den lokalen und regionalen Regierungen bzw. von der Bundesregierung drei Dollar bereitgestellt.

3 Dieser Ausdruck mit verschiedenen Bedeutungen wird hier im allgemeingültigen Sinn gebraucht; er umfasst Mitglieder einer ethnischen oder nationalen Gruppe, die Verbindungen zu ihrem Herkunftsland oder -gebiet aufrechterhalten. 
Diane Duclos stellt diesen Prozess in einer Fallstudie vor, die den Künstlern, Journalisten und Intellektuellen der irakischen Diaspora gewidmet ist. Über die Literaturzeitschrift Mesopotamia und virtuelle Cafés bedienen sich die Exiliraker des „Web“, um über den Wiederaufbau des irakischen Staates zu diskutieren. Wegen der Besetzung und der täglichen Gewalt im Lande bleibt die Beteiligung ihrer Mitbürger im Irak beschränkt. Jedoch sind sich Duclos und Meyer darüber einig, dass sich die virtuellen, finanziellen und realen Umlaufmethoden ablösen und ergänzen.

\section{Die Rolle der Diasporas}

Der transnationale Austausch hat das Bewusstwerden von der Rolle der Zugewanderten bei der Entwicklung ihres eigenen Landes, nicht nur in finanzieller, sondern auch in sozialer und politischer Hinsicht, erleichtert. Migration beruht auf einer kollektiven oder individuellen Entscheidung von Personen, die sich zur Auswanderung entschliessen und dafür die Folgen letztlich selbst tragen. Diese Erkenntnis hat die internationalen Organisationen und zahlreiche im Entwicklungsbereich tätige Nichtregierungsorganisationen immer häufiger dazu veranlasst, die menschlichen Ressourcen und die potenzielle Rolle der Migrantinnen und Migranten auf diesem Gebiet aufzuwerten.

Marc Antoine Pérouse de Montclos, der sich auf verschiedene, aus dem afrikanischen Kontext gegriffene Beispiele stützt, stellt fest, dass die Ausgewanderten Erfolgsmodelle bieten, zu neuen Vorstellungsweisen anregen, das Fortschrittsstreben fördern und zuweilen entscheidend zur Umverteilung der Macht im Herkunftsland beitragen. Gleichzeitig kann das Exil mit einer Neudefinition der Rollen zwischen Männern und Frauen einhergehen, was den Zugang der Mädchen zur Ausbildung erleichtert und es ihnen ermöglicht, sich den rückständigsten Traditionen zu entziehen. Doch gerade so, wie die Geldüberweisungen, die in Somalia sowohl zum Wiederaufbau kriegszerstörter Gebiete als auch zum Unterhalt bewaffneter Konflikte beitragen, kann der Beitrag der Diaspora sowohl die Demokratisierung und die Friedensverhandlungen fördern, als auch bestehende Spaltungen zwischen Klans oder Bevölkerungsgruppen noch vertiefen.

Diese gegensätzlichen, für das Emigrationsland gleichzeitig positiven und negativen Auswirkungen widerspiegeln sich in den Aussagen von Ibrahima Thioye, der Mauretanien Anfang der 1960er Jahre verliess und nach Frankreich ging, um seinem kranken Vater zu helfen. Thioye bedauert die Tatsache, dass die Sicht der Migranten - wie bei den meisten Menschen, wäre man versucht, hinzufügen - einzig auf ihre Familie, ihr Haus und ihr Dorf gerichtet ist, und dass sie Mühe haben, langfristig zu planen.

Diese Tendenz wird gemäss Pérouse de Montclos durch die wirtschaftliche und rechtliche Prekarität verstärkt, die das Los zahlreicher Migranten ist. Sie beschränkt ihren Einfluss, wie dies auch von Janine Dahinden und Joëlle Moret aufgrund einer diachronischen Analyse der serbischen und kosovo-albanischen Migrantenvereine in der Schweiz festgestellt wird. Indem die Autorinnen die Entwicklung des transnationalen Engagements dieser Organisationen nachzeichnen, stellen sie fest, dass humanitäre Vereine - in denen vor allem Frauen tätig sind - und politische Vereinigungen, die zur Zeit der bewaffneten Konflikte 
in Serbien und im Kosovo sehr aktiv waren, sich aufgelöst haben oder sich derzeit auf Integrationsprobleme in der Schweiz konzentrieren. Jedoch vermerken die beiden Autorinnen auch, dass die staatlichen Behörden und Institutionen im Herkunftsland wie im Aufnahmeland - ungeachtet des sozioökonomischen Profils der Migrantinnen und Migranten - einen entscheidenden Einfluss auf die Ausrichtung und die Aktivitäten der Migrantenvereine ausüben.

\section{Zu besseren Rahmenbedingungen}

Wenn es von nun an unerlässlich scheint, Migrantinnen und Migranten als vollwertige Akteure zu betrachten, warnen die Autoren Meyer und Pérouse de Montclos, wie auch die Autorinnen Dahinden und Moret vor der Versuchung, die Diasporas zu quasi natürlichen Entwicklungsagenten zu erklären, die es allein ermöglichen würden, mit dem mutmasslichen Scheitern der internationalen Entwicklungszusammenarbeit fertig zu werden. Die verschiedenen Beiträge weisen übereinstimmend darauf hin, dass eine fruchtbare Einbeziehung der Zugewanderten in den Entwicklungsprozess ohne eine Unterstützung der Herkunfts- und Zielländer nicht denkbar ist. Angesichts der zunehmenden Komplexität der Migrationsbewegungen erscheinen einseitige Ansätze zur Regulierung der Migration als immer unwirksamer. Auch wenn die Staaten weiterhin argwöhnisch an ihrer Souveränität in diesem Bereich festhalten, werden sie sich dennoch nach und nach der Tatsache bewusst, dass nur eine auf internationaler Ebene abgestimmte Aktion imstande ist, konstruktive Lösungen zu finden, die sowohl für die Herkunfts-, Transit- und Zielländer als auch für die Migrantinnen und Migranten selbst annehmbar sind.

Die Prämissen einer sinnvollen Politikgestaltung in diesem Bereich sind unter anderem Gegenstand des Artikels von Denis Drechsler und Jason Gagnon. Wirtschaftlich betrachtet so betonen die Autoren, sei es oft schwierig, die Beiträge der Migration von den für die wirtschaftliche und soziale Entwicklung eines Landes nachteiligen Folgen klar zu unterscheiden, da die Auswirkungen je nach Kontext und Migrationszyklus variieren. Der durch die Abwanderung qualifizierter Fachkräfte verursachte Humankapitalverlust kann sich beispielsweise in einen Gewinn umschlagen, wenn die Emigrationsaussichten die jungen Leute dazu bringen, sich auszubilden, wie Patricia A. Santo Tomas am Beispiel der Philippinen veranschaulicht. Der Fall der Philippinen ist umso bemerkenswerter, als die philippinische Regierung gegenüber der Migration eine sektorübergreifende Politik entwickelt hat, die sich von der Ausbildung vor der Abreise, über die Unterstützung durch Sozialattachés in den Zielländern, bis hin zu Wiedereingliederungsmassnahmen nach der Rückkehr erstreckt. Die Vorgehensweise im Hinblick auf den Abschluss bilateraler Abkommen für einen verbesserten sozialen Schutz der Migranten verdient ebenfalls, erwähnt zu werden. Trotzdem bleibt noch viel zu tun, da die philippinischen Überseearbeitende (Overseas Filipino Workers), von denen heute 60 Prozent Frauen sind, trotzdem häufig unter sehr prekären Arbeitsbedingungen (Zeitarbeit und/oder Schwarzarbeit) tätig sind.

Drechsler und Gagnon, wie auch Santo Tomas betonen die - von politischen Kreisen nicht immer zur Kenntnis genommene - Tatsache, dass die interkontinentale Migration weiterhin ein integraler Bestandteil der künftigen globalen Entwicklung sein wird. Unter der Voraussetzung, dass der Austausch und die 
Mobilität beiderseits gefördert werden, können qualifizierte Migranten wie auch weniger qualifizierte Arbeitskräfte durch Investitionen, Handel sowie durch die Übermittlung von Kompetenzen und sozialem wie politischem Know-how zur Entwicklung beitragen. In diesem Sinne empfehlen die Autoren den OECDStaaten, ihre Sektorpolitiken besser zu koordinieren, aber auch mehr Partnerschaften mit den Herkunftsstaaten einzugehen und die Diaspora in die Entwicklungspolitik mit einzubeziehen.

\section{Migration und Entwicklungszusammenarbeit: eine ambivalente Beziehung}

Die Idee, die Migranten - direkt oder über Organisationen - an die wirtschaftliche Entwicklung ihres Dorfs oder ihrer Region anzubinden, steht auch im Mittelpunkt des in Frankreich geprägten Begriffs „,codéveloppement“. Jérôme Audran zeigt auf, wie die traditionell in sicherheitspolitischen Anliegen verwurzelte, französische Migrationspolitik sich nach und nach für Entwicklungsfragen geöffnet hat. Die Gründung des französischen Ministeriums für Immigration, Integration, nationale Identität und solidarische Entwicklung (Ministère de l'Immigration, de l'Intégration, de l'Identité nationale et du Codéveloppement) im Jahr 2007 drückt den Willen von Nicolas Sarkozy aus, eine starke Bindung zwischen Entwicklungspolitik und Migrationssteuerung zu schaffen. Nachdem der Autor die wichtigsten historischen Etappen des Aufkommens des Konzepts „codéveloppement“ nachgezeichnet hat, bewertet er die positiven und negativen Seiten der Instrumentalisierung der Entwicklungszusammenarbeit zur Bekämpfung der illegalen Immigration. Trotz dieser Ambivalenz kommt der Autor zum Schluss, dass ,das codéveloppement die Möglichkeit bietet, die Migrationsproblematik unter dem Gesichtspunkt gemeinsamer Interessen anzugehen und so die Nord-Süd-Zusammenarbeit zu stärken“.

\section{Die Situation in der Schweiz}

Der letzte Teil dieses Dossiers befasst sich mit der Politik der Schweiz im Bereich der Beziehungen zwischen Migration und Entwicklung. Gianni D'Amato beschreibt zuerst die Geschichte der Migrations- und Asylpolitik der Schweiz und analysiert dann deren Entwicklung. Diese Entwicklung ist geprägt durch eine je nach dem Qualifikationsniveau der Personen differenzierte Zulassungspolitik (Öffnung für hoch qualifizierte Personen und für Personen aus der Europäischen Union, ehemaliges Saisonnierstatut für weniger qualifizierte Personen und Zuwanderungsstop für wenig qualifizierte Personen). Der Autor hebt die zunehmend restriktive Dynamik der Politik der Schweiz hervor.

Der nationale politische Kontext der Verschärfung des Asylgesetzes und des Ausländergesetzes hat die Bemühungen um eine breiter angelegte Migrationsdebatte nicht verhindert. Die Schweiz hat die Reflexion auf internationaler Ebene vorangetrieben, um den Dialog zwischen Herkunfts- und Aufnahmeländern zu verbessern. Auf nationaler Ebene wurden seit den 1980er Jahren bedeutende Anstrengungen für eine bessere interdepartementale Koordination zwischen den von der Migration betroffenen Ämtern unternommen. 
Pietro Mona beschreibt die Etappen, die zu dieser interdepartementalen Zusammenarbeit führten, und unterstreicht die Notwendigkeit eines ganzheitlichen Ansatzes (whole-of-government approach) für eine kohärentere Politik. Er verweist darauf, dass die Schweiz mit der Lancierung der Berner Initiative im Jahr 2001 einen wichtigen Impuls für eine verstärkte internationale Zusammenarbeit im Migrationsbereich gab, der zur Schaffung der Weltkommission für internationale Migration (Global Commission on International Migration) führte. In ihrem im Oktober 2005 vorgelegten Bericht befasst sich die Kommission prioritär mit der Beziehung zwischen Migration und Entwicklung, und die Reflexion wird im Rahmen des 2006 eingesetzten Hochrangigen Dialogs über internationale Migration und Entwicklung (High-Level Dialogue on International Migration and Development) fortgeführt.

Während die Schweiz in den internationalen Prozessen sehr aktiv ist, hat sie zuvor jedoch zahlreiche Debatten auf nationaler Ebene - namentlich im Rahmen der Verwaltung - geführt. Den Anstoss hierfür bildeten die Herausforderungen, die sich infolge der Asylmigration aus dem früheren Jugoslawien ergaben. Dieses Gebiet war vor dem Ausbruch der Kriege ein grosses Arbeitskräftereservoir gewesen. Die Erfahrung der Mitte der 1990er Jahre eingeführten Rückkehrhilfe veranschaulicht die Bedeutung einer zwischen den verschiedenen Politikbereichen (Aussenpolitik, Entwicklungspolitik, Asyl- und Ausänderpolitik, etc.) abgestimmten Zusammenarbeit.

Eric Kaser und Saskia Schenker liefern eine detaillierte Beschreibung der Funktionsweise der Rückkehrhilfe, welche ein wichtiges Instrument der schweizerischen Aussenpolitik im Migrationsbereich ist. Die individuelle Rückkehrhilfe für die Person, die in ihr Land zurückkehren muss, wird durch gezielte Unterstützungsmassnahmen (Ausbildung, Hilfe im Gesundheitsbereich) ergänzt. Vor allem aber geht sie mit einer Strukturhilfe vor Ort in einher, die von der Direktion für Entwicklung und Zusammenarbeit (DEZA) im Rahmen von Länderprogrammen erbracht wird. Die Rückkehrhilfe wird von mehreren Bundesämtern, namentlich vom Bundesamt für Migration und von der DEZA in gemeinsamer Abstimmung entwickelt. Sie kam in zehn Jahren über 65000 Menschen zugute, davon 40000 Personen im Balkangebiet (vor allem in Bosnien-Herzegowina und im Kosovo).

Hinsichtlich einer globaleren Ausrichtung sieht der Artikel 100 des Anfang 2008 in Kraft getretenen neuen Ausländergesetzes vor, dass der Bundesrat bilaterale Partnerschaften im Migrationsbereich mit anderen Staaten fördert. Therese Liechti und Monica Budowski untersuchen die Bedeutung dieser Abkommen. Gemäss den Autorinnen sind drei Aspekte bei der Vorbereitung der Migrationspartnerschaften wichtig. Zum einen muss die Art der Zusammenarbeit jedem der Partner erlauben, seine eigenen Interessen $\mathrm{zu}$ vertreten. Zum anderen muss man sich über die Unterscheidung zwischen ,erwünschter“ und „unerwünschter“ Migration einigen: Die Prioritäten ändern sich je nachdem, ob man sich auf die Stärkung der Kontrolle zur Verhinderung unerwünschter Migration oder auf Massnahmen zur Förderung des positiven Migrationspotenzials für die Entwicklung eines Landes konzentriert. Ferner ist die interdepartementale Zusammenarbeit eine Voraussetzung und zugleich der Schlüssel für den Aufbau erfolgreicher Migrationspartnerschaften. Die Autorinnen Kaser und Schenker verweisen darauf, dass die Erfahrungen mit den im Balkangebiet 
durchgeführten Rückkehrhilfeprogrammen bei der Errichtung der ersten Migrationspartnerschaft der Schweiz nützlich sein können.

Gérard Perroulaz untersucht in seinem Beitrag die Beziehungen zwischen der Politik der Entwicklungszusammenarbeit und der Migrationspolitik in der Schweiz. Die schweizerische Entwicklungszusammenarbeit räumt der Migrationsthematik im Diskurs grosse Bedeutung ein, und verschiedene Bundesämter haben sich für einen Dialog auf internationaler Ebene eingesetzt. Hingegen war es schwieriger, die Thematik in die Praxis umzusetzen, ausser im Bereich der Nothilfe. Auch wenn in einigen Schwerpunktländern der DEZA Synergien möglich waren, sind die Massnahmen der schweizerischen Entwicklungszusammenarbeit weiterhin hauptsächlich auf die Nothilfe und auf die Bekämpfung der negativen Auswirkungen der Migration ausgerichtet. Der Artikel schlägt einige Ansätze zur Förderung einer aktiveren Rolle der Entwicklungszusammenarbeit und zur Stärkung der positiven Auswirkungen auf die Entwicklung der Herkunftsländer der Migranten vor.

Christoph Wehrli führt einige Gründe an, die das Zögern der schweizerischen Entwicklungszusammenarbeit erklären können, sich in der Ausarbeitung von Programmen zur Zusammenarbeit im Migrationsbereich zu engagieren. Das Migrationsphänomen wird zwar regelmässig in den Botschaften des Bundesrates über die Entwicklungszusammenarbeit angeführt, jedoch waren Entwicklungsfachleute wie auch die politischen Kreise wenig gewillt, einen Teil des Entwicklungshilfebudgets zur Regelung von Asylfragen zu verwenden. Da die Schweizer Entwicklungszusammenarbeit befürchtet, zur Eindämmung und zur Verhütung der Migrationsflüsse instrumentalisiert zu werden, und da es ihr widerstrebt, vom Schwerpunktziel der Hilfe für die Ärmsten abzuweichen, hat sie sich bislang im Migrationsbereich kaum eingesetzt.

Dennoch haben Nichtregierungsorganisationen (NRO) der Entwicklungszusammenarbeit vor kurzem eine Reflexion zur Stärkung der Synergien zwischen Migration und Entwicklung eingeleitet. Yanik Marguerat und Christelle Genoud beschreiben in ihrem Beitrag die derzeit im Rahmen der Fédération genevoise de coopération in diesem Bereich unternommenen Reflexionen. Sie stellen aber fest, dass es noch wenig Projekte der Solidarität und der Entwicklungszusammenarbeit gibt, die einen Dialog zwischen NRO des Nordens, Empfängergemeinschaften des Südens und Migrantenverbänden vorsehen.

$$
* * *
$$

Die Tatsache, dass die Migration ein integraler Bestandteil der sozioökonomischen Entwicklung unserer Gesellschaften ist, und dass diese fast immer von internen oder internationalen Migrationsbewegungen abhängig ist, ist von der Forschung bereits seit langem nachgewiesen, wie Ronald Skeldon vor kurzem anmerkte ${ }^{4}$. Die verschiedenen Beiträge dieses Jahrbuchs stellen einige der vielfältigen Facetten des Nexus zwischen Migration und Entwicklung vor. Andere, nicht weniger wichtige Aspekte konnten in diesem Rahmen nicht behandelt werden. Dabei denken wir insbesondere an die spezifische Dynamik der

4 „International Migration as a Tool in Development Policy: A Passing Phase ?“, Population and Development Review, Bd. 34, Nr. 1, März 2008, S. 1-18. 
Zwangsmigration in Krisengebieten. Die Vertreibung von Teilen der Bevölkerung und ihre Betreuung in Lagern mit mehreren Zigtausend Menschen stellen nicht nur grosse sozioökonomische und ökologische Herausforderungen dar, sondern werfen auch Sicherheits- und Stabilitätsfragen für die betroffenen Länder auf, von denen einige zu den ärmsten der Welt gehören. Um solchen Krisensituation zu begegnen, die bisweilen mehrere Jahrzehnte andauern, ist es erforderlich, die Folgen der humanitären Einsätze, der Migrations- und der Entwicklungspolitik besser zu kennen. Dabei machen zahlreiche internationale Akteure die Entscheidungsinstanzen auch auf die Auswirkungen des Klimawandels und der Umweltzerstörung aufmerksam, die ebenfalls zu unfreiwilligen Wanderungsbewegungen führen können.

Die Zunahme und die neue Dynamik der Migration von Frauen würden allein für sich ein Dossier in Anspruch nehmen; so entscheidend sind ihre Folgen für die Entwicklung. Man denke dabei nur an die zahlreichen Migrantinnen, die sich in den Grossstädten des Nordens und Südens um Kinder und Betagte kümmern und für den Unterhalt ganzer Familien sorgen, während ihre eigenen Kinder im Herkunftsland von anderen Familienmitgliedern aufgezogen werden. Obgleich diese Frauen zuweilen über gute berufliche Qualifikationen verfügen, arbeiten sie oft illegal, während die industrialisierten Länder weltweit um die „besten Köpfe“ wetteifern. Auch wenn die problematischsten Aspekte der neuen weiblichen Migration, wie beim Menschenhandel, in der Forschung Beachtung fanden, wurde das mit der autonomen Mobilität von Frauen verbundene Potenzial bislang noch wenig erkundet.

Um es den Leserinnen und Lesern zu erlauben, sich mit Themen, die hier nicht ausführlich erörtert werden konnten, vertraut zu machen, haben wir einige Eintrittsportale ins Internet ausgewählt, die auf spezielle Websites hinweisen. Die von Chantal Hebeisen und Martine Schaer unter der Rubrik Hintergrundinformation vorgeschlagenen Websites stellen keine umfassende Liste dar, sondern bieten eine Auswahl politischer, nichtstaatlicher oder wissenschaftlicher Institutionen in der Schweiz und weltweit.

Zum Abschluss dieser Einführung können wir darauf hinweisen, dass das erneute Interesse für die Beziehungen zwischen Migration und Entwicklung immerhin den Vorteil hat, in Erinnerung zu rufen, dass Migration in der gesamten Menschheitsgeschichte mit Entwicklung einhergegangen ist. Heute wie gestern wäre es illusorisch, die internationale Migration vollständig steuern oder unterbinden zu wollen. Es gilt im Gegenteil, internationale Migration als Realität zur Kenntnis zu nehmen, und anzuerkennen, dass sie ein wichtigen Vorteil für die Entwicklung der Herkunftsgebiete und - nicht zu vergessen der Aufnahmeländer darstellen kann. Die im vorliegenden Jahrbuch präsentierten Artikel heben indes die Komplexität der Zusammenhänge zwischen beiden Phänomenen hervor. Trotz der politisch motivierten Diskurse, die eher zur Verwirrung beitragen, ergibt sich aus der Lektüre der verschiedenen Beiträge eine grundlegende Feststellung: Migration wird niemals eine Patentlösung für die Entwicklung der Länder des Südens sein, wie auch die Entwicklung dieser Länder die Migrationsbewegungen nicht abwenden kann. Diese Feststellungen laufen einigen Botschaften zuwider, die mehr oder minder explizit versuchen, das Image von zwei Politikfeldern (Migration und Entwicklungszusammenarbeit) aufzuwerten, die in den letzten Jahren zahlreichen Kritiken ausgesetzt 
waren. Es ist somit kein Zufall, wenn diese Thematik ihre Rückkehr in den Mittelpunkt des Interesses politischen Kreisen und internationalen Organisationen verdankt.

Dennoch muss festgestellt werden, dass sich das fragliche Handlungsfeld für eine direkte und voluntaristische Politikgestaltung wenig eignet, aus den bereits erwähnten Gründen, und weil es zahlreichen Spannungsfeldern und Einflüssen vielfältiger Akteure ausgesetzt ist. Diese Feststellung legt nahe, dass die „Zweckallianz“ zwischen beiden Politikbereichen niemals zu einer spontanen „Liebesheirat" führen wird. Dies will natürlich nicht besagen, dass die zahlreichen derzeit laufenden oder bereits erfolgten Debatten und Forschungen über die Annäherung zwischen Migration und Entwicklung vergeblich seien. Ganz im Gegenteil: Sie tragen zum unerlässlichen Verständnis des Zusammenspiels beider Phänomene und der Wechselwirkung zwischen involvierten Partnern bei. Umso besser auch, wenn die Migrantinnen und Migranten fortan als vollwertige Akteure anerkannt werden, deren Interessen - genau so wie jene der Herkunftsund der Aufenthaltsländer - zu berücksichtigen sind. In Anbetracht der präsentierten Ergebnisse läuft eine Instrumentalisierung des einen Politikfeldes im Dienst des anderen Gefahr, Wirkungen auszulösen, die den angestrebten Wirkungen mittel- oder langfristig zuwiderlaufen. Was den Handlungsbedarf betrifft, so geht es darum, Foren für die Verständigung und Koordination zu schaffen, die den Beteiligten genügend Spielraum lassen, um sinnvolle Strukturen und Rahmenbedingungen zur Mobilitätsförderung auszuarbeiten. Es ist somit anzunehmen, dass Erfolg und Nachhaltigkeit einer solchen Zweckallianz eher auf einer breiten Palette von Massnahmen beruhen werden, die mit allen Betroffenen im Sinne eines soft policy-Ansatzes gut abgesprochen werden, als in einer spektakulären politischen Wende. Gerade dies dürfte es der Migration erlauben, entwicklungsfördernde Wirkungen zu entfalten. 